\title{
O conhecimento dos Agentes Comunitários de Saúde sobre o calendário vacinal infantil
}

Community Health Agents' knowledge about the infant vaccination calendar

El conocimiento de los Agentes Comunitarios de Salud sobre el calendario de vacunación infantil

Recebido: 27/05/2021 | Revisado: 06/06/2021 | Aceito: 07/06/2021 | Publicado: 22/06/2021

Andrea Oliveira da Silva de Almeida ORCID: https://orcid.org/0000-0003-0073-5966 Universidade do Estado do Pará, Brasil E-mail: deiaoliver19@yahoo.com.br

Angélica Menezes Bessa Oliveira ORCID: https://orcid.org/0000-0001-7644-4538 Universidade do Estado do Pará, Brasil E-mail: angelbssa@hotmail.com

Ana Caroline Guedes Souza Martins ORCID: https://orcid.org/0000-0002-8447-6828 Universidade do Estado do Pará, Brasil E-mail: carolguedes.devs@hotmail.com

Nadia Pinheiro da Costa

ORCID: https://orcid.org/0000-0001-6550-366X Universidade do Estado do Pará, Brasil E-mail: enfnadya@gmail.com

Thamyres da Silva Martins

ORCID: https://orcid.org/0000-0002-6988-5404 Universidade do Estado do Pará, Brasil E-mail: thamyresms@yahoo.com.br Nivea Moreira Leite Pereira

ORCID: https://orcid.org/0000-0002-6414-0243 Universidade do Estado do Pará, Brasil E-mail: enfenivea.pereira@gmail.com

Bruno Rafael Ribeiro de Almeida ORCID: https://orcid.org/0000-0002-6080-5826 Instituto Federal do Pará, Brasil

E-mail: bruno.almeida@ifpa.edu.br

Marcelle de Nazaré Lima de Sousa ORCID: https://orcid.org/0000-0001-6550-366X Escola Superior da Amazônia, Brasil E-mail: sousa_marcelle@yahoo.com.br

Rosângela Lima da Silva

ORCID: https://orcid.org/0000-0003-2000-4343 Universidade do Estado do Pará, Brasil E-mail: rosangela.silva@uepa.br

Renata Coelho Rodrigues Noronha ORCID: https://orcid.org/0000-0001-5014-4991 Universidade Federal do Pará, Brasil E-mail: renatarcrn@gmail.com

Flávia dos Santos Tavares ORCID: https://orcid.org/0000-0003-0941-5811 Universidade Federal do Pará, Brasil E-mail: flaviatavares.ft.77@gmail.com

Luan Felipe da Silva Frade ORCID: https://orcid.org/0000-0001-9706-4817 Universidade Federal do Pará, Brasil E-mail: luffrade@gmail.com

Gal Caroline Alho Lobão ORCID: https://orcid.org/0000-0002-2803-4224

Instituto Evandro Chagas, Brasil E-mail: enfa.gal@gmail.com

Sarah Jacqueline Costa do Lago ORCID: https://orcid.org/0000-0002-1714-1569 Hospital Universitário João de Barros Barreto, Brasil E-mail: sarahlago@gmail.com 


\author{
Emanuela Chaves da Silva \\ ORCID: https://orcid.org/0000-0001-9338-1006 \\ Universidade Federal do Pará, Brasil \\ E-mail: manuchavesmc@gmail.com \\ Priscila Pinheiro de Miranda \\ ORCID: https://orcid.org/0000-0001-7866-5086 \\ Faculdade Pan Amazônica, Brasil \\ E-mail: pripinheiro46@gmail.com \\ Camila Cristina Girard Santos \\ ORCID: https://orcid.org/0000-0003-3159-7041 \\ Universidade do Estado do Pará, Brasil \\ E-mail: camilagirard@ hotmail.com \\ Taynnara de Oliveira do Espirito Santo Cunha \\ ORCID: https://orcid.org/0000-0001-9659-615X \\ Instituto Federal do Pará, Brasil \\ E-mail: tayoliveiraenf@gmail.com \\ Ana do Socorro Maia de Moraes \\ ORCID: https://orcid.org/0000-0003-0076-3780 \\ Universidade do Estado do Pará, Brasil \\ E-mail: amaiademoraes@yahoo.com.br \\ Márcia Bitar Portella \\ ORCID: https://orcid.org/0000-0002-4602-7618 \\ Universidade do Estado do Pará, Brasil \\ E-mail: marciabitar@gmail.com
}

\title{
Resumo
}

Este estudo objetiva identificar o conhecimento dos Agentes Comunitários de Saúde (ACS) sobre o calendário vacinal infantil de crianças até cinco anos. Trata-se de uma pesquisa quantitativa, descritiva, do tipo antes e depois, com amostra de 25 ACS de duas Unidades Básicas de Saúde de Ananindeua-Pará. A média de idade dos ACS é de 40 anos; sexo feminino (64\%); 76\% verifica o cartão de vacina da criança e dá orientações às mães; $96 \%$ afirmou que verificar o cartão de vacina da criança faz parte do seu trabalho; $100 \%$ realiza visitas domiciliares periódicas às crianças de sua área, 88\% têm crianças com vacinas atrasadas na sua área. Quanto aos ACS se sentirem seguros para orientar as mães sobre vacinas, observou-se aumento do índice do pré para pós capacitação (26,1\%-73,9\%). Ao serem questionados se receberam treinamento no pré e pós capacitação, o índice subiu de 78,3\% para 100\%. Quanto ao nível de conhecimento sobre vacinas em crianças até 5 anos, houve aumento no pré e pós capacitação nos conceitos "ótimo" (0\%-8,7\%), "bom" (21,7\%- 43,5\%), "regular" (39,1\%-43,5\%) e diminuição do conceito "insuficiente" (39,1\%-4,3\%). Houve aumento da taxa média de acertos dos ACS no pré e pós capacitação $(61,9 \%-81,1 \%)$ com diferença entre as taxas de acertos nas fases pré e pós de 19,18\%. Conclui-se que há a necessidade de investimento em qualificação profissional dos ACS por meio da educação permanente, a fim de ampliar os conhecimentos sobre calendário vacinal. Palavras-chave: Agentes Comunitários de saúde; Imunização; Educação permanente.

\begin{abstract}
This study aims to identify the knowledge of Community Health Agents (CHA) about the childhood vaccination schedule for children up to five years old. This is a quantitative, descriptive, before-and-after research, with a sample of $25 \mathrm{CHA}$ from two Basic Health Units in Ananindeua-Pará. The average age of CHA is 40 years old; female (64\%); $76 \%$ check the child's vaccination card and provide guidance to mothers; $96 \%$ said that checking the child's vaccination card is part of their job; $100 \%$ carry out periodic home visits to children in their area, $88 \%$ have children with delayed vaccinations in their area. As for the CHA feeling safe to guide mothers about vaccines, there was an increase in the rate from pre to post training (26.1\%-73.9\%). When asked if they received training in pre and post training, the rate rose from $78.3 \%$ to $100 \%$. As for the level of knowledge about vaccines in children up to 5 years of age, there was an increase in pre and post training in the concepts "great" (0\%-8.7\%), "good" (21.7\%-43.5\%), "regular" (39.1\%-43.5\%) and a decrease in the "insufficient" concept (39.1\%-4.3\%). There was an increase in the average hit rate of the $\mathrm{CHA}$ in the pre and post training $(61.9 \%-81.1 \%)$ with a difference between the hit rates in the pre and post phases of $19.18 \%$. It is concluded that there is a need for investment in professional qualification of the CHA through permanent education, in order to expand their knowledge about the vaccination calendar.
\end{abstract}

Keywords: Community health agent; Immunization; Permanent education.

\section{Resumen}

Este estudio tiene como objetivo identificar el conocimiento de los Agentes de Salud Comunitarios (CHA) sobre el calendario de vacunación infantil para niños de hasta cinco años. Se trata de una investigación cuantitativa, descriptiva, de antes y después, con una muestra de 25 CHA de dos Unidades Básicas de Salud de Ananindeua-Pará. La edad promedio de CHA es de 40 años; femenino (64\%); El 76\% revisa la cartilla de vacunación del niño y brinda orientación a las madres; El 96\% dijo que revisar la cartilla de vacunación del niño es parte de su trabajo; El 100\% realiza visitas domiciliarias periódicas a los niños de su área, el 88\% tiene niños con vacunación tardía en su área. En 
cuanto a que las CHA se sintieron seguras para orientar a las madres sobre las vacunas, hubo un aumento en la tasa desde antes hasta después de la capacitación (26,1\%-73,9\%). Cuando se les preguntó si habían recibido formación previa y posterior a la formación, la tasa aumentó del 78,3\% al $100 \%$. En cuanto al nivel de conocimiento sobre vacunas en niños hasta los 5 años de edad, hubo un incremento en la pre y post formación en los conceptos "genial" (0\%-8,7\%), "bueno" (21,7\% -43,5\%), "Regular" (39,1\%-43,5\%) y una disminución del concepto de "insuficiente" $(39,1 \%-4,3 \%)$. Hubo un aumento en la tasa de aciertos promedio de los CHA en el pre y post entrenamiento $(61,9 \%-$ $81,1 \%$ ) con una diferencia entre las tasas de aciertos en las fases pre y post del $19,18 \%$. Se concluye que existe la necesidad de invertir en la calificación profesional de la CHA através de la educación permanente, con el fin de ampliar sus conocimientos sobre el calendario de vacunación.

Palabras clave: Agentes de salud comunitarios; Inmunización; Educación permanente.

\section{Introdução}

A reforma do modelo assistencial em saúde no Brasil, tomando por base o que Movimento Sanitário preconizava, desencadeou ao final da década de 1980 na criação do Sistema Único de Saúde (SUS), pautado nos princípios da universalidade, integralidade, equidade, descentralização, regionalização e participação social (Maciel et al., 2020).

Foi por meio da luta política da reforma sanitária que os movimentos populares, profissionais da saúde, associação de moradores e outros atores sociais construíram uma agenda de discussão positiva de direitos sociais que culminou no SUS (Secco et al., 2020).

Nesse contexto, a Atenção Primária à Saúde (APS) foi criada pelo SUS com o objetivo de melhorar o acesso aos serviços de saúde e potencializar a resolutividade das ações. É a porta de entrada com ampla resolutividade aos níveis secundário e terciário, os quais se complementam. Esse novo sistema teve por premissa a promoção da saúde, acesso do cidadão ao sistema de saúde e qualidade de vida, tendo a integralidade, universalidade e equidade como norteadores da organização dos serviços (Godoi et al., 2018).

No processo de construção do SUS, ao longo dos últimos 32 anos, destaca-se a implantação do Programa de Agentes Comunitários de Saúde (PACS), a partir de 1991, e o Programa de Saúde da Família (PSF), criado em 1994 que, posteriormente, passa a ser considerado como Estratégia de Saúde da Família onde a categoria profissional do Agente Comunitário de Saúde (ACS) foi vinculado ao PSF, para atuar nas unidades básicas e constituir o elo entre a comunidade e os serviços de saúde (Maciel et al., 2020).

A Estratégia Saúde da Família é preferencialmente a porta de entrada do indivíduo e caracteriza-se por um conjunto de ações de saúde no âmbito individual, familiar e coletivo, que ofertam a promoção e proteção da saúde, prevenção de agravos, diagnóstico, tratamento, reabilitação, redução de danos, cuidados paliativos e vigilância em saúde, além disso deve atender às demandas e necessidades de saúde da população (Brasil, 2017).

Antes das atualizações da Política Nacional da Atenção Básica (PNAB), as visitas domiciliares periódicas às famílias de sua área eram a maior parte do trabalho desses profissionais e, nos casos em que detectassem algum problema, encaminhavam a questão aos outros profissionais da equipe (Secco et al., 2020).

A partir da nova PNAB (2017) as atividades dos ACS e dos Agentes de Combate às Endemias (ACE) passaram por uma integração, uma vez que a Atenção Primária e a Vigilância em Saúde devem unidas identificar problemas de saúde nos territórios e planejar estratégias de intervenção clínica e sanitária efetivas (Secco et al., 2020).

Dentre as atribuições dos ACS que são de suma importância para o bom funcionamento da APS estão a identificação de fatores socioeconômicos, culturais e ambientais que possam interferir na saúde da população de sua área; planejamento de ações estratégicas juntamente com a equipe; acompanhamento da comunidade por meio de visitas domiciliares; execução de atividades educativas individuais e coletivas e formação de grupos operativos (Godoi et al., 2018).

A definição do ACS como peça fundamental para o sistema de saúde traz a necessidade de reconhecimento e valorização de seu papel na ESF. A capacitação de forma efetiva desses atores é uma ação que pode contribuir para essa 
valorização uma vez que é insuficiente e deficitária, não os preparando devidamente para atuar nos problemas que deparam durante o exercício de seu trabalho. É notória a necessidade de investir cada vez mais na capacitação desses profissionais, a fim de proporcionar maior eficiência nas funções de promoção da saúde e prevenção de agravos na comunidade adstrita pela UBS (Godoi et al., 2018).

Três aspectos devem ser levados em conta ao discutir o processo de formação ou qualificação de recursos humanos dos ACS: o perfil do profissional a ser capacitado; sua qualificação e necessidades de formação; e, quais as competências devem ser desenvolvidas ou adquiridas no processo educacional (Tomaz, 2002).

Capacitar de forma efetiva os ACS em situações recorrentes sobre condições de saúde, dentre as quais, o calendário vacinal infantil, tendo em vista as constantes mudanças ocorridas anualmente, trará benefícios relevantes na prevenção de doenças imunopreveníveis contribuindo para a melhoria das coberturas vacinais, quadro preocupante em que vive o país.

É por meio das visitas rotineiras dos ACS em todas as residências da área de abrangência que a situação de saúde das crianças pode ser monitorada e a realização de orientações específicas para cada caso, diminuindo, assim, o atraso vacinal e, consequentemente, aumentando a cobertura vacinal desde público (Bujes, 2012).

O Programa Nacional de Imunização (PNI) criado pelo Ministério da Saúde em 1973 tem como objetivo coordenar as ações de imunizações e controle de doenças de imunopreviníveis através do alcance de 100\% de coberturas vacinais (Brasil, 2012).

A erradicação de morbidades como poliomielite e varíola tornou-se possíveis de forma exitosa graças ao intenso trabalho de imunização disseminada em crianças da primeira infância no Brasil e esforço de profissionais do SUS para o alcance deste objetivo, dentre eles, o ACS. No entanto, mesmo com todos os esforços dos gestores e equipes de saúde, o atraso vacinal é um problema recorrente em diversos municípios do Brasil. Estudos revelam que mais de 24 milhões de crianças ainda não têm acesso a esta importante intervenção de saúde, contribuindo para milhões de mortes infantis evitáveis em países periféricos. Os esforços para melhorar a cobertura vacinal nestes países são fundamentais para cumprimento dos Objetivos de Desenvolvimento Sustentável de reduzir a mortalidade infantil, conforme prevê a Organização das Nações Unidas -Agenda 2030. (Assad et al., 2020).

A imunização constitui-se em cuidados primários de saúde e propicia a resposta quase que imediata na prevenção de doenças, mas, para que seja de qualidade e tenha eficácia, é importante que o indivíduo receba todas as doses necessárias do calendário vacinal nas datas apropriadas, principalmente durante o primeiro ano de vida (Bujes, 2012).

Oportunidades perdidas de vacinação acontecem todos os dias nas unidades de saúde, portanto, todos os participantes de uma equipe de saúde devem estar atentos e preparados para intervir ativamente na busca dessas crianças com vacinas em atraso (Bujes, 2012).

Nesse contexto, propõe-se a Educação Permanente em Saúde (EPS) como movimento que produz processos de educação no próprio espaço de trabalho, pois os trabalhadores de saúde necessitam constantemente refletir sobre suas práticas, avaliá-las nas perspectivas individuais e coletivas, avançando no conhecimento e na direção de maior qualificação das ações e serviços de saúde desenvolvidos para atender usuários/cidadãos (Assad et al., 2020).

Faz-se necessário criar estratégias de promoção de ações de EPS para as equipes de saúde, especialmente aquelas segundo as normas do Programa Nacional de Imunização (PNI). Dessa forma, percebe-se a importância de ações educativas acerca do tema imunização, por ser uma ferramenta e instrumento de grande valia para promoção e prevenção, em todos os níveis de atenção, mas é principalmente na ESF que se busca fortalecimento e embasamento de ações para melhoria da qualidade de vida da população assistida. Apesar de a vacinação ser tema de predomínio da equipe de enfermagem, ao contextualizá-lo com outros atores, o dirigimos na perspectiva da EPS, visando principalmente reflexão crítica profissional ante temas referentes à imunização (Assad et al., 2020). 
Ademais, o planejamento mostra-se como importante ferramenta para o desenvolvimento de competências não sendo possível sua realização de forma isolada, estando arraigado à seu caráter dinâmico, uma vez que a realidade do cotidiano do trabalho está em constante transformação (Cosme \& Valente, 2020).

Deste modo, esta pesquisa tem por objetivo identificar o conhecimento dos Agentes Comunitários de Saúde sobre o calendário vacinal infantil de crianças até cinco anos.

\section{Metodologia}

Esta pesquisa caracteriza-se como de intervenção descritiva do tipo antes e depois, com abordagem quantitativa. Para Fleury e Werlang (2017), a pesquisa descritiva objetiva compreender o fenômeno e descrever a sua distribuição em uma determinada população. Exige do investigador uma série de informações sobre o que deseja pesquisar. Entende-se que a pesquisa descritiva é abrangente, permite analisar o problema em relação aos aspectos sociais, econômicos, políticos e percepções de diferentes grupos, comunidade, e também é utilizada para compreensão de diferentes comportamentos.

Enquanto que a pesquisa quantitativa é aquela em que se coletam e analisam dados quantitativos sobre variáveis. Dessa forma, este tipo de pesquisa é capaz de identificar a natureza profunda das realidades, seu sistema de relações, sua estrutura dinâmica. Ela também pode determinar a força de associação ou correlação entre variáveis, a generalização e objetivação dos resultados por meio de uma mostra que faz inferência a uma população. Além do estudo da associação ou correlação, a pesquisa quantitativa também pode, ao seu tempo, fazer inferências causais que explicam por que as coisas acontecem ou não de uma forma determinada (Esperón, 2017).

Participaram da pesquisa 25 ACS que atuam nas UBSs do bairro Icuí, que é composta por três Estratégias de Saúde da Família (ESF), e na UBS do bairro Cidade Nova 6, composta por quatro ESF, localizadas no município de Ananindeua, Pará. Em ambos os locais são realizados serviços de visita domiciliar pela equipe multiprofissional, consultas, entre outros serviços previstos pelo Ministério da Saúde, como curativos, atendimento odontológico, triagem e sala de vacina.

Foram incluídos na pesquisa os ACS de ambos os sexos que estivessem em vínculo atual com as unidades de saúde. Foram excluídos da pesquisa os ACS cuja admissão aconteceu no período inferior a um ano ou que estavam afastados por quais motivos, tais como licença, férias, dentre outros.

A coleta de dados ocorreu em três etapas, durante 4 dias consecutivos (dois dias para cada unidade de saúde), nas próprias dependências das unidades de saúde. Na primeira etapa, os participantes preencheram um questionário com questões fechadas, envolvendo o conteúdo sobre o conhecimento do calendário vacinal, contraindicações, reações adversas e mitos sobre vacinas e dados socioeconômicos. Esta etapa finalizou-se em apenas um dia. Na segunda etapa os ACS foram capacitados em um treinamento com metodologias ativas de aprendizagem onde após leitura do material fornecido e discussão de casos em grupos de 4 e 5 pessoas um calendário vacinal foi confeccionado por eles. A capacitação dos participantes aconteceu com a utilização da problematização como estratégia de ensino, com estudos de caso, baseados em suas experiências, associada à exposição tradicional do conteúdo. Na terceira etapa o mesmo teste da primeira etapa foi aplicado a fim de se investigar se houve aumento de conhecimentos no público-alvo.

O questionário foi aplicado entre seus pares não participantes da pesquisa preliminarmente como um estudo piloto a fim de verificar a sua viabilidade, no entanto, como não houve dificuldade em seu preenchimento, o questionário foi utilizado na íntegra.

Para a análise dos dados foi aplicada a estatística descritiva, através do Teste Qui-quadrado de Pearson para tendência (variáveis nominais), Teste t de Student para comparação de médias (variáveis numéricas). Utilizou-se a Análise de Regressão Logística para verificar os efeitos das variáveis sociodemográficas sob a probabilidade de acerto, antes e após o treinamento.

A pesquisa teve aprovação pelo Comitê de Ética e Pesquisa do Centro de Ciências Biológicas e da Saúde (CCBS) da 
Universidade do Estado do Pará (UEPA), sob parecer n0 3.029.283/2018 e atendeu aos preceitos éticos da Resolução $n^{\circ}$ 466/2012 do Conselho Nacional de Saúde.

\section{Resultados e Discussão}

Verifica-se na Tabela 1 que a maioria dos Agentes Comunitários de Saúde submetidos a avaliação de conhecimento sobre o calendário vacinal infantil possuem idade entre 34 e 43 anos (10; 40\%) e idade média de 40 anos $(\mu=39,68)$, variando com desvio padrão de \pm 10 anos $( \pm \sigma=10,29)$ (figura 1), sexo feminino (16;64\%), 10 (40\%) agentes possuem tempo de profissão entre 2 e 6 anos e 10 (40\%) possuem tempo de profissão entre 17 e 21 anos; O tempo médio de profissão dos ACS é de 11 anos $(\mu=10,76)$, variando com desvio padrão de \pm 8 anos $( \pm \sigma=7,71)$

Além disso, observa-se que todos os ACS $(25 ; 100 \%)$ participantes da pesquisa declararam que realizam visitas às crianças da área de atuação, 19 (76\%) agentes afirmaram que verificam o cartão de vacina da criança e dão orientações às mães, 24 (96\%) agentes acham que verificar o cartão de vacina da criança faz parte do seu trabalho, todos os ACS (25; 100\%) participantes da pesquisa declararam que alguma mãe ou pai já perguntou ao agente sobre o cartão de vacina de seu filho e a maioria dos agentes $(22 ; 88 \%)$ afirmaram que em sua área tem crianças com vacina atrasada.

Tabela 1: Caracterização dos Agentes Comunitários de Saúde submetidos a avaliação de conhecimento sobre o calendário vacinal infantil. Ananindeua, Pará, Brasil, 2020.

\begin{tabular}{|c|c|c|c|}
\hline Caracterização & $\mathbf{n}=\mathbf{2 5}$ & $\%$ & P-Valor ${ }^{(1)}$ \\
\hline \multicolumn{4}{|l|}{ Faixa Etária } \\
\hline $24-33$ & 7 & $28,0 \%$ & \multirow{4}{*}{$0.233^{\text {ns }}$} \\
\hline $34-43$ & 10 & $40,0 \%$ & \\
\hline $44-53$ & 5 & $20,0 \%$ & \\
\hline $54-63$ & 3 & $12,0 \%$ & \\
\hline \multicolumn{4}{|l|}{ Sexo } \\
\hline Feminino & 16 & $64,0 \%$ & \multirow{2}{*}{$0.162^{\text {ns }}$} \\
\hline Masculino & 9 & $36,0 \%$ & \\
\hline \multicolumn{4}{|c|}{ Tempo de profissão (anos) } \\
\hline $2-6$ & 10 & $40,0 \%$ & \\
\hline $7-11$ & 3 & $12,0 \%$ & \\
\hline $12-16$ & 2 & $8,0 \%$ & \\
\hline $17-21$ & 10 & $40,0 \%$ & $0.028^{*}$ \\
\hline \multicolumn{4}{|c|}{ 1- realiza visita às crianças da área? } \\
\hline Sim & 25 & $100,0 \%$ & \\
\hline Não & 0 & $0,0 \%$ & $0.000 *$ \\
\hline \multicolumn{4}{|c|}{ 2- verifica o cartão de vacina da criança e dá orientações às mães? } \\
\hline Sim & 19 & $76,0 \%$ & \\
\hline Não & 6 & $24,0 \%$ & $0.009^{*}$ \\
\hline \multicolumn{4}{|c|}{ 3- acha que verificar o cartão de vacina da criança faz parte do seu trabalho? } \\
\hline Sim & 24 & $96,0 \%$ & \\
\hline Não & 1 & $4,0 \%$ & $0.000^{*}$ \\
\hline \multicolumn{4}{|c|}{ 4- alguma mãe ou pai já perguntou à você sobre o cartão de vacina de seu filho? } \\
\hline Sim & 25 & $100,0 \%$ & \\
\hline Não & 0 & $0,0 \%$ & $0.000 *$ \\
\hline \multicolumn{4}{|c|}{ 6- Em sua área tem crianças com vacina atrasada? } \\
\hline Sim & 22 & $88,0 \%$ & \\
\hline Não & 3 & $12,0 \%$ & $0.000 *$ \\
\hline $\begin{array}{l}\text { Nota: Os resultado } \\
\text { (1) Teste Qui-quadr } \\
\text { *Valores Significa } \\
\text { Interpretação do te } \\
\mathrm{H}_{0} \text { : As frequências } \\
\text { Ha: As frequências } \\
\text { Decisão: Como o v } \\
\text { Ha. } \\
\text { Fonte: Protocolo de }\end{array}$ & $\begin{array}{l}\text { para os di } \\
\text { a os difer } \\
\text { e signific }\end{array}$ & itar a hipó & aceitar a hipótese alternativa \\
\hline
\end{tabular}


Estes resultados corroboram com o estudo de Santos et al. (2020), que afirma que quanto ao conhecimento sobre o conteúdo da Caderneta de Saúde da Criança (CSC), 99,1\% (n=108) referiram conhecer o instrumento, e 91,6\% ( $\mathrm{n}=98)$ assinalaram usá-la rotineiramente. Já em relação aos questionamentos sobre conhecimento prévio dos itens que compõem a caderneta da criança, o item vacinação foi assinalado por 99,1\% $(n=106)$. Portanto, identificou-se que a maioria dos ACS apresenta maior familiaridade com determinados aspectos da CSC como o acompanhamento do esquema vacinal e do ganho ponderal das crianças (Santos et al., 2020).

Estes resultados mostram que há uma valorização, por parte dos ACS, quanto ao item "vacinação" da Caderneta de Saúde da Criança, em detrimento a outros instrumentos como que compõe a caderneta. Em um estudo realizado em UBS de municípios de São Paulo, o esquema vacinal estava completamente preenchido em 97\% das CSC (Amorim et al., 2018). As evidências mostram que a maioria dos ACS afirmam que tinham conhecimento para orientar sobre a CSC, contudo referiram necessidade de capacitação sobre esse conteúdo (Santos et al., 2020).

Acredita-se que um dos fatores deste atraso das vacinas infantis seja em decorrência das dificuldades dos ACS em detectar quais vacinas estão em atraso e como realizar corretamente as orientações, justificando a necessidade de capacitações frequentes para atualização quanto as mudanças rotineiras que ocorrem no Calendário Vacinal do Programa Nacional de Imunizações (PNI).

Um dos trabalhadores que compõe a rede de APS no Brasil é o ACS, e uma das principais atividades desse profissional é a visita domiciliar, seguida da educação em saúde. Informações sobre vacinação são uma das atividades educativas mais realizadas por este profissional, por meio de orientações que costumam prestar às famílias (Santos et al., 2020).

Além disso, os ACS possuem algumas atribuições que são de suma importância para o bom funcionamento da APS como a identificação de fatores socioeconômicos, culturais e ambientais que possam interferir na saúde da população de sua área; planejamento de ações estratégicas juntamente com sua equipe de saúde; execução de atividades educativas individuais e coletivas e formação de grupos operativos (Musse, 2015).

Estes profissionais conhecem profundamente a realidade local (valores, linguagem, perigos, etc), bem como acarretam vivências e experiências para o interior da equipe de saúde e abrem novos caminhos no processo de intervenção (Guedes et al., 2014; Avelar, 2014).

O ACS é considerado peça fundamental para o sistema de saúde, portanto, faz-se necessário evidenciar que eles precisam ser reconhecidos e valorizados pelo papel que exercem na ESF. Contudo, as capacitações destinadas a esses atores é insuficiente e deficitária, não os preparando devidamente para atuar nos problemas que deparam durante o exercício de seu trabalho (Silva \& Gaíva, 2016).

Uma das características fundamentais em um ACS é a liderança natural na comunidade fundamentada na capacidade de se comunicar com as pessoas, para estimular a corresponsabilidade pela melhoria da qualidade de vida e saúde da população. Desta forma, os programas de capacitação devem ser elaborados de forma a terem como objetivo transformar os ACS em sujeitos proativos (Santos et al., 2020).

Na capacitação com o tema "O papel da ACS no Contexto da ESF" foi demonstrado que o trabalho desses agentes é uma extensão dos serviços de saúde na comunidade onde atuam por serem membros da população local. Segundo a comparação dos resultados obtidos nos questionários gerais pré e pós-capacitações, a percepção dos agentes em relação à importância de seu trabalho para o funcionamento da UBS sofreu alterações, sendo que antes das capacitações metade das agentes comunitárias consideravam-se "extremamente importante" para a equipe e após as capacitações a maioria consideravase "muito importante". Uma das hipóteses para tal resultado é que, com o decorrer do projeto, houve aproximação entre os ACS e os alunos obtendo-se, portanto, maior liberdade para serem sinceras nas respostas ao questionário. Outra possibilidade é 
que elas adquiriram melhor percepção e importância da equipe, considerando seu papel fundamental quando realizado em conjunto (Santos et al., 2020).

Desta forma, a Caderneta de Saúde da Criança (CSC) é considerada um instrumento de vigilância do crescimento e do desenvolvimento infantil, elaborado pelo Ministério da Saúde e preconizado para ser utilizada por todos os profissionais que assistem a criança, que devem realizar registro correto e completo das condições de saúde, inclusive da situação vacinal (Brasil, 2013).

Estudos evidenciam a precária utilização da CSC com elevado percentual de preenchimento inadequado, comprometendo, assim, a avaliação e o acompanhamento do crescimento e do desenvolvimento das crianças pelos profissionais de saúde (Almeida et al., 2016; Sociedade Brasileira de Pediatria, 2017).

A precária utilização da CSC está relacionada à diversos fatores como a carência e à fragilidade de registros, à dificuldade dos profissionais de saúde de perceberem a relevância do preenchimento, ao conhecimento deficiente por parte desses profissionais, além da carência de orientações às famílias (Lima et al., 2016).

A adequada utilização da caderneta pelas equipes de saúde possibilita maior valorização e apropriação do instrumento pela família, além de favorecer a adesão e a corresponsabilização pelas ações de vigilância da saúde de seus filhos (Almeida et al., 2016; Sociedade Brasileira de Pediatria, 2017).

O preenchimento do instrumento é um procedimento de baixa complexidade. A falha deste registro demonstra a fragilidade da assistência que não necessita de participação de pessoal com qualificação de nível superior (Costa et al., 2014).

No estudo de Santos (2011), em questionário aplicado previamente às capacitações, foram evidenciadas variações de opinião quanto a importância da CSC no desenvolvimento como ACS, no qual os resultados variaram entre "Muito Importante" até "Extremamente Importante". Após as capacitações, a percepção dessa importância se manteve, demonstrando que, possivelmente, a aplicação das capacitações reforçou a posição dos agentes quanto à importância da educação continuada em saúde. No entanto, as capacitações podem se refletir de forma distinta a cada ACS, tendo influência de alguns aspectos como conhecimento prévio acerca dos temas trabalhados, dedicação durante as capacitações e características individuais.

A Tabela 2 mostra que na fase pré-capacitação apenas $6(26,1 \%)$ ACS declararam se sentir seguros para orientar a mãe sobre vacinas da criança, essa frequência aumentou para 17 (73,9\%) ACS na fase pós-capacitação. O número de agentes que recebeu treinamento também aumentou na fase pós-capacitação, passando de 18 (78,3\%) ACS na fase pré, para 23 (100\%) ACS na fase pós-capacitação. Na fase pré-capacitação, nenhum ACS considerava ótimo o nível de seu conhecimento sobre vacinas em crianças até 5 anos, essa frequência aumentou para 2 (8,7\%) ACS. A frequência de ACS que acreditava possuir um conhecimento insuficiente diminuiu de $9(39,1 \%)$ para 1 (4,3\%) na fase pós-capacitação. 
Tabela 2: Distribuição dos agentes comunitários de saúde submetidos a avaliação de conhecimento sobre o calendário vacinal infantil, segundo a taxa de conhecimento e treinamento dos agentes, pré e pós capacitação. Ananindeua, Pará, Brasil, 2020.

\begin{tabular}{|c|c|c|c|c|c|}
\hline \multirow{2}{*}{ Variável } & \multicolumn{2}{|c|}{ Pré-Capacitação $(n=23)$} & \multicolumn{2}{|c|}{ Pós-Capacitação $(n=23)$} & \multirow{2}{*}{ P-Valor ${ }^{(1)}$} \\
\hline & $\mathbf{n}$ & $\%$ & $\mathbf{n}$ & $\%$ & \\
\hline \multicolumn{6}{|c|}{ 5- você se sente seguro para orientar a mãe sobre vacinas da criança? } \\
\hline Sim & 6 & $26,1 \%$ & 17 & $73,9 \%$ & \multirow{2}{*}{$0,003 *$} \\
\hline Não & 17 & $73,9 \%$ & 6 & $26,1 \%$ & \\
\hline \multicolumn{6}{|c|}{ 7- Já teve algum treinamento sobre vacinação? } \\
\hline Sim & 18 & $78,3 \%$ & 23 & $100,0 \%$ & \multirow{2}{*}{$0,049 *$} \\
\hline Não & 5 & $21,7 \%$ & 0 & $0,0 \%$ & \\
\hline \multicolumn{6}{|c|}{ 8- como considera o seu conhecimento sobre vacinas em crianças até 5 anos? } \\
\hline Ótimo & 0 & $0,0 \%$ & 2 & $8,7 \%$ & \multirow{4}{*}{$0,008 *$} \\
\hline Bom & 5 & $21,7 \%$ & 10 & $43,5 \%$ & \\
\hline Regular & 9 & $39,1 \%$ & 10 & $43,5 \%$ & \\
\hline Insuficiente & 9 & $39,1 \%$ & 1 & $4,3 \%$ & \\
\hline
\end{tabular}

Nota: Os resultados são baseados em linhas e colunas não vazias em cada subtabela mais interna.

(1) Teste Exato de Fisher para independência (p-valor<0.05).

*Valores Significativos; NS - Valores Não Significativos.

Interpretação do teste:

$\mathrm{H}_{0}$ : Não há relação significativa de dependência entre a variável da linha e a variável de coluna.

Ha: Há relação significativa de dependência entre a variável da linha e a variável de coluna.

Decisão: Como o valor de $p$ computado é menor que o nível de significância alfa $=0,05$, deve-se rejeitar a hipótese nula $\mathrm{H}_{0}$ e aceitar a hipótese alternativa Ha.

Fonte: Protocolo de pesquisa (2020).

Quanto aos ACS se sentirem seguros para orientar as mães sobre vacinas e se receberam treinamento, observou-se que houve aumento do índice do pré para pós capacitação. E quando questionados sobre o nível de seu conhecimento sobre vacinas em crianças até 5 anos, os resultados do pré e pós capacitação mostram que o conceito “ótimo" subiu de $0 \%$ para $8,7 \%$, "bom" subiu de 21,7\% para 43,5\%, "regular" subiu de 39,1\% para 43,5\% e "insuficiente" caiu de 39,1\% para 4,3\%. Estes dados demonstram os benefícios da capacitação que foi ministrada.

Corroborando com estes achados, a capacitação sobre o tema "Calendário de Vacinação e Interpretação do Cartão de Vacina" foi baseada no conteúdo da "Caderneta de Saúde da Criança” e "Calendário Nacional de Vacinação". Na autoavaliação da capacidade dos ACS por meio de questionário, a maioria sentiu-se "Pouco capacitada" em relação ao tema antes da capacitação. No entanto, após aplicação de tal tema, já se julgavam "Capacitada” para lidar com o tema. No questionário final, as alternativas "Sem capacidade" e "Extremamente capacitadas" não foram preenchidas por nenhum deles (Santos et al., 2020).

Na Figura 1 observa-se quanto a taxa média de acertos dos ACS no pré e pós capacitação, em que houve aumento de $61,9 \%$ para $81,1 \%$ e, portanto, a diferença encontrada entre as taxas de acertos obtidas nas fases pré e pós foi de $19,18 \%$. Ademais, constatou-se que as chances foram 12 vezes maiores de acertar as respostas na fase pós-capacitação, e a porcentagem total de acertos antes e após capacitação subiu de $62,4 \%$ para $82,8 \%$. 
Figura 1: Boxplot de comparação das taxas médias de acertos dos agentes comunitários de saúde submetidos a avaliação de conhecimento sobre o calendário vacinal infantil, por fases de avaliação. Ananindeua, Pará, Brasil, 2020.

\section{Boxplot de Acertos (\%)}

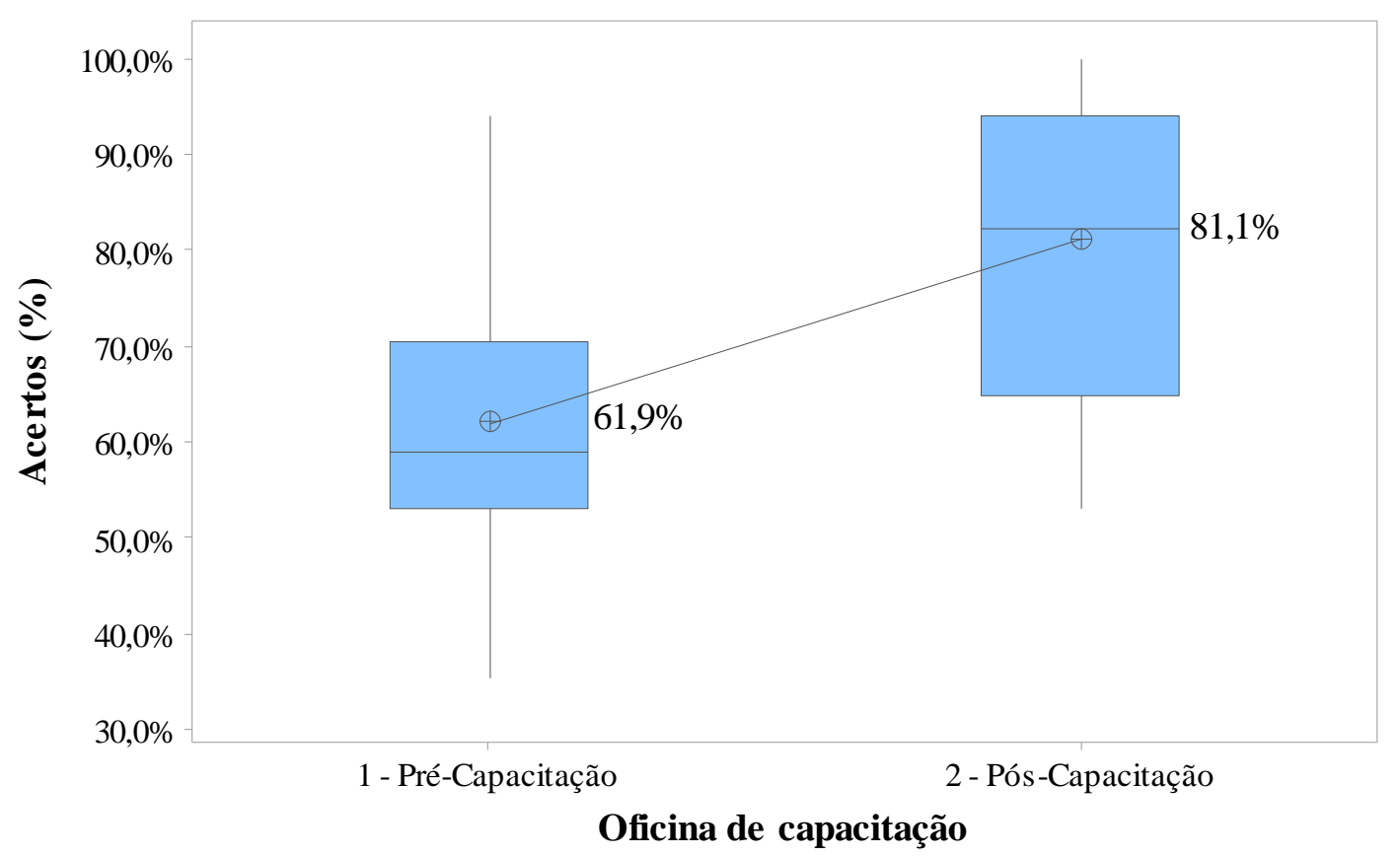

Fonte: Protocolo de pesquisa (2020).

O estudo de Martins et al. (2019) vem reforçar a importância da Educação Permanente (EP) para profissionais de saúde que atuam na imunização, sendo este considerado um desafio, visto que as alterações no calendário vacinal são frequentes., além dos mitos e verdades que permeiam este processo, como os ventos adversos, as dificuldades enfrentadas no cotidiano, o fluxo de profissionais, a rotatividade e a precarização da contratação, que prejudicam muito o trabalho na unidade de saúde, assim como no vínculo com o usuário (Martins et al., 2019).

Considera-se, ainda, a inovação e o aprimoramento tecnológico, bem como as situações cotidianas do trabalho em saúde e as particularidades que advêm do trabalho em equipe que demandam um permanente processo de educação dos profissionais (Assad et al., 2017).

Em consonância com o conceito adotado pelo Ministério da Saúde brasileiro, por meio da Política Nacional de Educação Permanente em Saúde (PNEPS), a EP pode ser entendida como um processo de aprendizagem-trabalho pautado em estratégias pedagógicas que se utilizam da aprendizagem significativa, com vistas a alcançar a transformação das práticas profissionais. A construção da EP advém dos problemas enfrentados na realidade, considerando os conhecimentos e as experiências que as pessoas já têm, bem como as necessidades de saúde das populações (Brasil, 2018).

Fortalecer a EP como norteadora de novas práticas que orientam a reflexão sobre o trabalho e a construção de processos de aprendizagem colaborativa e significativa é, consequentemente, fortalecer o SUS para ofertar ações coletivas com profissionais de saúde em permanente formação frente aos principais desafios identificados pelas equipes no cotidiano do trabalho (Brasil, 2014).

Como implicações para a prática, a EP é um campo que carece de investimentos no Brasil. Não se pode ser vista somente como ferramenta de organização do sistema de saúde ou estratégia para remodelar o processo de trabalho, com a realização de cursos ou ações educacionais pontuais, restrita a momentos formais instituídos. Ela deve ser entendida como 
dispositivo para mediar mudanças, permitindo aos sujeitos um processo de autoanálise no trabalho, além e por meio do trabalho como possibilidade de crescimento para lidar com o mundo (Campos, Sena \& Silva, 2017).

Mesmo sem ser visto, esse movimento de EP vai acontecendo como prática e com seus efeitos. Esse processo é constitutivo do próprio mundo do trabalho e vai ocorrendo sem que precise ser denominado como processo formativo, para ser, de fato, lugar de formação (Merhy, 2015).

Desta forma, os treinamentos, as capacitações e educação de profissionais para a imunização devem ocorrer rotineiramente na agenda da EP, não só quando se tem inserção de novas vacinas no calendário ou quando vai ter alguma campanha ou alguma mudança. Assim, faz-se necessário a decisão em equipe das necessidades de EP relacionadas a imunização, para se determinar o foco do conteúdo a ser discutido, tornando-se este um processo democrático, com a participação de toda a equipe da UBS. Estudos mostram que este processo ocorre de maneira reversa, ou seja, não se baseia nas necessidades e problemas identificados no cotidiano de trabalho, indo na contramão do que é proposto pela PNEPS (Martins et al., 2019).

A EP, em consonância com o descrito na PNEPS, deve ser pautada por metodologias problematizadoras, dialogadas e participativas, em que o profissional se apresente ativamente no processo de ensino-aprendizagem, com vistas à melhoria de suas práticas, bem como o contínuo aperfeiçoamento (Cunha et al., 2014).

Evidenciou-se que não há relação de dependência entre a faixa etária (tabela 3), sexo (tabela 4) e tempo de profissão (tabela 5) dos ACS e a taxa média de acertos na pré e pós-capacitação sobre o calendário vacinal infantil. Porém, destaca-se que ocorreu aumento na taxa média de acertos na pós-capacitação para todas as faixas etárias, ambos os sexos e todas as faixas de tempo de profissão, com destaque para a maior taxa média de acertos que ocorreu na faixa de 12 a 16 anos de profissão.

Tabela 3: Taxas médias de acertos dos agentes comunitários de saúde na pré e pós-capacitação sobre o calendário vacinal infantil, por faixa etária. Ananindeua, Pará, Brasil, 2020.

\begin{tabular}{|c|c|c|c|c|c|c|c|}
\hline \multicolumn{2}{|c|}{ Faixa Etária } & \multirow[t]{2}{*}{$\mathbf{N}$} & \multirow{2}{*}{$\begin{array}{l}\text { Média } \\
55,5 \%\end{array}$} & \multirow{2}{*}{$\begin{array}{c}\text { DesvPad } \\
12,6 \%\end{array}$} & \multicolumn{2}{|c|}{ IC de $95 \%$} & \multirow[t]{2}{*}{ P-Valor ${ }^{(1)}$} \\
\hline \multirow{4}{*}{$\begin{array}{c}\text { Pré- } \\
\text { capacitação }\end{array}$} & $24-33$ & & & & $43,9 \%$ & $67,0 \%$ & \\
\hline & $34-43$ & 9 & $66,7 \%$ & $14,4 \%$ & $56,5 \%$ & $76,9 \%$ & \multirow{3}{*}{$0.522^{\mathrm{ns}}$} \\
\hline & $44-53$ & 4 & $61,8 \%$ & $19,5 \%$ & $46,5 \%$ & $77,1 \%$ & \\
\hline & $54-63$ & 3 & $62,8 \%$ & $12,3 \%$ & $45,1 \%$ & $80,4 \%$ & \\
\hline \multicolumn{8}{|c|}{ DesvPad Combinado $=0,146209$} \\
\hline \multirow{4}{*}{$\begin{array}{c}\text { Pós- } \\
\text { capacitação }\end{array}$} & $24-33$ & 7 & $77,3 \%$ & $18,4 \%$ & $65,4 \%$ & $89,2 \%$ & \multirow{4}{*}{$0.340^{\text {ns }}$} \\
\hline & $34-43$ & 9 & $88,2 \%$ & $8,8 \%$ & $77,7 \%$ & $98,7 \%$ & \\
\hline & $44-53$ & 4 & $73,5 \%$ & $20,1 \%$ & $57,8 \%$ & $89,3 \%$ & \\
\hline & $54-63$ & 3 & $78,4 \%$ & $14,8 \%$ & $60,2 \%$ & $96,6 \%$ & \\
\hline
\end{tabular}

DesvPad Combinado $=0,150592$

Nota: Os resultados são baseados em linhas e colunas não vazias em cada subtabela mais interna.

(1) Teste de Tukey (p-valor $<0.05$ ).

*Valores Significativos; NS - Valores Não Significativos.

Interpretação do teste:

$\mathrm{H}_{0}$ : As médias observadas não diferem significativamente entre os grupos etários.

Ha: As frequências observadas diferem significativamente para os diferentes grupos etários.

Decisão: Como o valor de $p$ computado é menor que o nível de significância alfa $=0,05$, deve-se rejeitar a hipótese nula $\mathrm{H}_{0}$ e aceitar a

hipótese alternativa Ha.

Fonte: Protocolo de pesquisa (2020). 
Tabela 4: Taxas médias de acertos dos agentes comunitários de saúde na pré e pós-capacitação sobre o calendário vacinal infantil, por sexo. Ananindeua, Pará, Brasil, 2020.

\begin{tabular}{|c|c|c|c|c|c|c|c|}
\hline \multicolumn{2}{|c|}{ Sexo } & \multirow{2}{*}{$\begin{array}{l}\mathbf{N} \\
4\end{array}$} & \multirow{2}{*}{$\begin{array}{l}\text { Média } \\
65,1 \%\end{array}$} & \multirow{2}{*}{$\begin{array}{c}\text { DesvPad } \\
13,2 \%\end{array}$} & \multicolumn{2}{|c|}{ IC de $95 \%$} & \multirow{2}{*}{$\begin{array}{c}\text { P-Valor } \\
0.148^{\text {ns }}\end{array}$} \\
\hline Pré- & Fem & & & & $57,6 \%$ & $72,6 \%$ & \\
\hline capacitaçâo & Masc & 3 & $55,9 \%$ & $15,4 \%$ & $45,6 \%$ & $66,2 \%$ & \\
\hline \multicolumn{8}{|c|}{ DesvPad Combinado = 0,146209 } \\
\hline \multirow{2}{*}{$\begin{array}{c}\text { Pós- } \\
\text { capacitação }\end{array}$} & Fem & 4 & $85,9 \%$ & $14,2 \%$ & $78,4 \%$ & $93,4 \%$ & $0.035 *$ \\
\hline & Masc & 3 & $72,1 \%$ & $13,6 \%$ & $61,8 \%$ & $82,4 \%$ & \\
\hline
\end{tabular}

DesvPad Combinado $=0,150592$

Nota: Os resultados são baseados em linhas e colunas não vazias em cada subtabela mais interna.

(1) Teste de Tukey (p-valor $<0.05$ ).

*Valores Significativos; NS - Valores Não Significativos.

Interpretação do teste:

$\mathrm{H}_{0}$ : As médias observadas não diferem significativamente entre os grupos etários.

Ha: As frequências observadas diferem significativamente para os diferentes grupos etários.

Decisão: Como o valor de $p$ computado é menor que o nível de significância alfa $=0,05$, deve-se rejeitar a hipótese nula $\mathrm{H}_{0}$ e aceitar a hipótese alternativa Ha.

Fonte: Protocolo de pesquisa (2020).

Tabela 5: Taxas médias de acertos dos agentes comunitários de saúde na pré e pós-capacitação sobre o calendário vacinal infantil, por tempo de profissão (anos). Ananindeua, Pará, Brasil, 2020.

\begin{tabular}{|c|c|c|c|c|c|c|c|}
\hline \multicolumn{2}{|c|}{ Tempo de profissão (anos) } & \multirow{2}{*}{$\begin{array}{l}\mathbf{N} \\
10\end{array}$} & \multirow{2}{*}{$\begin{array}{l}\text { Média } \\
54,7 \%\end{array}$} & \multirow{2}{*}{$\begin{array}{c}\text { DesvPad } \\
10,8 \%\end{array}$} & \multicolumn{2}{|c|}{ IC de $95 \%$} & \multirow[t]{2}{*}{ P-Valor ${ }^{(1)}$} \\
\hline \multirow{4}{*}{$\begin{array}{c}\text { Pré- } \\
\text { capacitação }\end{array}$} & $2-6$ & & & & $45,8 \%$ & $63,6 \%$ & \\
\hline & $7-11$ & 3 & $64,7 \%$ & $11,8 \%$ & $48,4 \%$ & $81,0 \%$ & \multirow{3}{*}{$0.142^{\mathrm{ns}}$} \\
\hline & $12-16$ & 2 & $76,5 \%$ & $25,0 \%$ & $56,5 \%$ & $96,4 \%$ & \\
\hline & $17-21$ & 8 & $66,2 \%$ & $14,7 \%$ & $56,2 \%$ & $76,1 \%$ & \\
\hline \multicolumn{8}{|c|}{ DesvPad Combinado $=0,146209$} \\
\hline \multirow{4}{*}{$\begin{array}{c}\text { Pós- } \\
\text { capacitação }\end{array}$} & $2-6$ & 10 & $\mathbf{7 5 , 9 \%}$ & $14,8 \%$ & $66,6 \%$ & $85,1 \%$ & \multirow{4}{*}{$0.101^{\mathrm{ns}}$} \\
\hline & $7-11$ & 3 & $92,2 \%$ & $13,6 \%$ & $75,3 \%$ & $109,1 \%$ & \\
\hline & $12-16$ & 2 & $100,0 \%$ & $0,0 \%$ & $79,3 \%$ & $120,7 \%$ & \\
\hline & $17-21$ & 8 & $78,7 \%$ & $14,0 \%$ & $68,3 \%$ & $89,0 \%$ & \\
\hline
\end{tabular}

DesvPad Combinado $=0,139882$

Nota: Os resultados são baseados em linhas e colunas não vazias em cada subtabela mais interna.

(1) Teste de Tukey ( $\mathrm{p}$-valor<0.05).

*Valores Significativos; NS - Valores Não Significativos.

Interpretação do teste:

$\mathrm{H}_{0}$ : As médias observadas não diferem significativamente entre os grupos etários.

Ha: As frequências observadas diferem significativamente para os diferentes grupos etários.

Decisão: Como o valor de $p$ computado é menor que o nível de significância alfa $=0,05$, deve-se rejeitar a hipótese nula $\mathrm{H}_{0}$ e aceitar a hipótese alternativa Ha.

Fonte: Protocolo de pesquisa (2020).

Na Tabela 6, evidenciou-se que tanto aqueles que alegaram que já tiveram algum treinamento sobre vacina $(42,1 \%)$ como aqueles que não tiveram (50\%), em sua maioria, consideram o seu conhecimento sobre vacinas em crianças até 5 anos de maneira regular. 
Tabela 6: Distribuição dos agentes comunitários de saúde na avaliação de conhecimento sobre o calendário vacinal infantil, segundo a relação entre as questões de aspectos gerais relacionadas ao trabalho. Ananindeua, Pará, Brasil, 2020.

\begin{tabular}{|c|c|c|c|c|c|c|c|}
\hline \multirow{3}{*}{$\begin{array}{c}\text { 8- como considera o seu } \\
\text { conhecimento sobre } \\
\text { vacinas em crianças até } \\
5 \text { anos? }\end{array}$} & \multicolumn{4}{|c|}{ 7- Já teve algum treinamento sobre vacinação? } & \multirow{2}{*}{\multicolumn{2}{|c|}{ Total }} & \multirow{3}{*}{$\begin{array}{c}\text { P- } \\
\text { Valor }^{(1)} \\
\end{array}$} \\
\hline & \multicolumn{2}{|c|}{ Sim } & \multicolumn{2}{|c|}{ Não } & & & \\
\hline & $\mathrm{n}$ & $\%$ & $\mathrm{n}$ & $\%$ & $\mathrm{n}$ & $\%$ & \\
\hline Bom & 5 & $26,3 \%$ & 0 & $0,0 \%$ & 5 & $20,0 \%$ & $0.201^{\mathrm{ns}}$ \\
\hline Regular & 8 & $42,1 \%$ & 3 & $50,0 \%$ & 11 & $44,0 \%$ & \\
\hline Insuficiente & 6 & $31,6 \%$ & 3 & $50,0 \%$ & 9 & $36,0 \%$ & \\
\hline Total & 19 & $\mathbf{7 6 , 0 \%}$ & 6 & $24,0 \%$ & 25 & $100,0 \%$ & \\
\hline
\end{tabular}

Fonte: Autores.

Com relação à sensação de segurança do ACS para orientar a mãe sobre vacinas da criança e alguns aspectos gerais relacionadas ao trabalho (Tabela 7), verificou-se que 100\% dos ACS realizam visitas as crianças da área, porém a maioria (68\%) não se sente seguro para orientar a mãe sobre vacinas da criança. A maioria verifica o cartão de vacina da criança e dá orientações às mães $(76 \%)$, porém uma proporção maior de ACS não se sente seguro para orientar a mãe sobre vacinas da criança (44\%). A maioria acha que verificar o cartão de vacina da criança faz parte do seu trabalho (96\%), porém uma proporção maior de ACS não se sente seguro para orientar a mãe sobre vacinas da criança (64\%). Todos os agentes já foram questionados por alguma mãe ou pai sobre o cartão de vacina do filho (100\%), porém a maioria (68\%) não se sente seguro para orientar a mãe sobre vacinas da criança.

Tabela 7: Distribuição dos agentes comunitários de saúde na avaliação de conhecimento sobre o calendário vacinal infantil, segundo a relação entre as questões de aspectos gerais relacionadas ao trabalho. Ananindeua, Pará, Brasil, 2020.

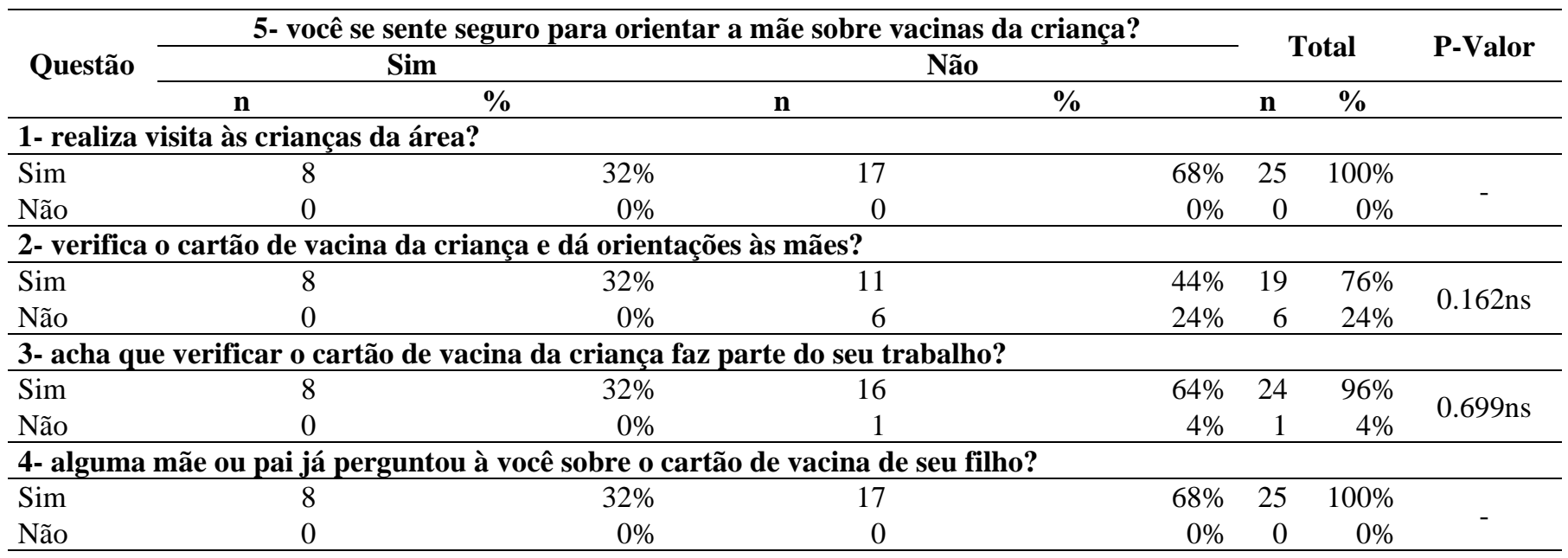

Nota: Os resultados são baseados em linhas e colunas não vazias em cada subtabela mais interna.

(1) Teste Exato de Fisher para independência (p-valor $<0.05$ ).

*Valores Significativos; NS - Valores Não Significativos.

Interpretação do teste:

$\mathrm{H}_{0}$ : Não há relação significativa de dependência entre a variável da linha e a variável de coluna.

Ha: Há relação significativa de dependência entre a variável da linha e a variável de coluna.

Decisão: Como o valor de $p$ computado é menor que o nível de significância alfa $=0,05$, deve-se rejeitar a hipótese nula $\mathrm{H}_{0}$ e aceitar a hipótese alternativa Ha.

Fonte: Protocolo de pesquisa (2020).

Na Tabela 8 observa-se as 17 afirmações sobre o calendário de vacinas de crianças vigente do Ministério da Saúde (questões 9 à 20 e 23), além de assertivas sobre mitos e verdades (questões 21, 22, 24 e 25) onde os participantes marcaram apenas uma alternativa (verdadeira ou falsa), conforme exposto a seguir: 
Tabela 8: Percentual de acertos nas afirmações específicas. Ananindeua, Pará, Brasil, 2020.

\begin{tabular}{|c|c|c|c|c|c|}
\hline \multirow{2}{*}{ Afirmação } & \multirow{2}{*}{ Vacina } & \multicolumn{2}{|c|}{ Antes da oficina } & \multicolumn{2}{|c|}{ Após a oficina } \\
\hline & & Acertos & $\%$ & Acertos & $\%$ \\
\hline 9 & BCG/hepatite B & 21 & $91,3 \%$ & 23 & $100 \%$ \\
\hline 20 & Hepatite B & 10 & $43,5 \%$ & 17 & $73,9 \%$ \\
\hline 10 & Pentavalente & 17 & $73,9 \%$ & 23 & $100 \%$ \\
\hline 11 & Pentavalente & 11 & $47,8 \%$ & 18 & $78,3 \%$ \\
\hline 12 & Rotavírus (VORH) & 17 & $73,9 \%$ & 18 & $78,3 \%$ \\
\hline 13 & Pneumocócica 23 & 09 & $39,1 \%$ & 17 & $73,9 \%$ \\
\hline 14 & Meningocócica AC & 17 & $73,9 \%$ & 21 & $91,3 \%$ \\
\hline 15 & Pólio oral (VOP) & 23 & $100 \%$ & 23 & $100 \%$ \\
\hline 16 & VIP/VOP & 13 & $56,5 \%$ & 15 & $65,2 \%$ \\
\hline 17 & Hepatite A & 10 & $43,5 \%$ & 14 & $60,9 \%$ \\
\hline 18 & Tríplice viral & 11 & $47,8 \%$ & 17 & $73,9 \%$ \\
\hline 19 & Febre amarela & 15 & $65,2 \%$ & 22 & $96 \%$ \\
\hline 23 & DTP & 15 & $65,2 \%$ & 17 & $73,9 \%$ \\
\hline 21 & $\begin{array}{l}\text { Mitos sobre administração simultânea de } \\
\text { vacinas }\end{array}$ & 10 & $43,5 \%$ & 18 & $78,3 \%$ \\
\hline 22 & Mito sobre vacina e criança gripada & 14 & $60,9 \%$ & 23 & $100 \%$ \\
\hline 24 & $\begin{array}{c}\text { Mito sobre alergias e administração de } \\
\text { vacinas. }\end{array}$ & 19 & $83,6 \%$ & 19 & $83,6 \%$ \\
\hline 25 & $\begin{array}{l}\text { Mito sobre diarreia e administração de } \\
\text { vacinas. }\end{array}$ & 12 & $52,17 \%$ & 19 & $83,6 \%$ \\
\hline & 17 afirmações & 244 & $62,4 \%$ & 324 & $82,8 \%$ \\
\hline
\end{tabular}

Fonte: Autores.

Observou-se que a porcentagem total de acertos antes da oficina foi 62,4\% (244 acertos). O bom percentual de acertos pode estar associado ao treinamento recebido anteriormente, que segundo o questionário 78,3\% receberam treinamentos anteriores sobre vacinas. Além disso, constatou-se que houve aumento de acertos quanto às afirmações, em que após o treinamento obteve-se um resultado de 82,8\% (324 acertos) após a realização da oficina.

Estes achados reforçam a necessidade de capacitações frequentes que motivem as ações de educação em saúde por parte dos ACS, a fim de dar embasamento teórico para sua prática profissional, conforme constatado por Maciazeki-Gomes et al. (2016), que afirma que a educação em saúde desempenhada pelo ACS é de fundamental importância para que se alcance um nível satisfatório de saúde de uma comunidade, pois conhecendo-se os principais agravos predominantes em uma área, e mesmo ao promover o conhecimento pessoal, é possível obter mudança nos paradigmas da saúde

Os personagens em contato com essa realidade e diretamente influenciado e afetado por ela são os ACS, que ao se depararem com essas diversas situações, precisam de um suporte dos demais membros da equipe de saúde e dos gestores, para evitar a fragilização de suas ações. Fica evidente a importância desse trabalhador e ao mesmo tempo o contraste com a pouca qualificação investida neles (Maciazeki-Gomes et al., 2016).

De acordo com Bomfim et al. 2017, o ACS ainda sofre com a falta de conhecimento sobre suas atribuições e o grau da magnitude delas na comunidade, associando tal fato a uma deficiência na realização de capacitações dos profissionais de saúde e até na implementação da política de educação permanente. Assim, o ACS como potencial educador, atua na prevenção de doenças e promoção de saúde, requerendo qualificação constante do seu trabalho (Maciazeki-Gomes et al., 2016).

\section{Conclusão}

A presente pesquisa possibilitou reflexões importantes acerca dos conhecimentos dos Agentes Comunitários de Saúde sobre o calendário vacinal infantil de crianças até cinco anos, bem como descrever o perfil sociodemográfico dos ACS e Capacitar/atualizá-los sobre imunização de acordo com as normas do PNI. 
A APS foi criada pelo SUS com o objetivo de melhorar o acesso e potencializar a resolutividade das ações. É o primeiro nível de atenção e a porta de entrada aos níveis secundário e terciário, os quais se complementam. Esse sistema tem como principal objetivo a promoção da saúde, com consequente acesso do cidadão ao sistema de saúde e qualidade de vida garantidos, tendo a integralidade, universalidade e equidade como norteadores da organização dos serviços. O ACS é o profissional da APS que exerce papel de "elo" entre equipe e comunidade, pois eles devem residir na área de atuação, o que proporciona uma maior proximidade com o cotidiano das famílias adscritas da ESF.

Além disso, a fim de proporcionar uma melhor assistência à comunidade, eles necessitam ser capacitados rotineiramente para realizar visitas domiciliares na área adstrita produzindo dados capazes de dimensionar os principais problemas de saúde em determinada área.

Há a necessidade de investimento em qualificação profissional dos ACS por meio da educação permanente em saúde, a fim de ampliar os conhecimentos em sua área, melhorar a qualidade dos serviços prestados e promover maior eficiência nas funções de promoção da saúde e prevenção de agravos na comunidade adstrita pela UBS.

Dessa maneira, espera-se que os ACS sejam capazes de orientar a população corretamente sobre vacinação infantil, bem como sobre as doenças e as consequências que podem ocorrer caso ocorram falhas neste processo de monitoramento da situação vacinal. Acredita-se que por meio da capacitação realizada tenha ocorrido uma melhora significativa quanto aos conhecimentos adquirido pelos profissionais ACS, bem como a partir das atividades lúdicas que foram realizadas possa ter aumentado o grau de comprometimento e senso de trabalho em equipe, levando a um aumento do estímulo para o trabalho na comunidade, já que este é ator importante da educação da população.

Outrossim, estudos futuros podem ser realizados para se conhecer a realidade de outros cenários de saúde, assim como a criação de tecnologias educacionais que podem ser elaboradas com vistas a melhorar a qualidade do trabalho dos ACS.

\section{Referências}

Almeida, A. C. et al. (2016). Use of a monitoring tool for growth hand development in Brazilian children: systematic review. Rev Paul Pediatr. Jan-Mar; $34(1), 122-31$.

Avelar, J. M. F. (2014). O Agente Comunitário de Saúde e a Educação Permanente em Saúde. 38 f. TCC (Graduação) Curso de Curso de Especialização em Atenção Básica em Saúde da Família, Universidade Federal de Minas Gerais, Lagoa Santa.

Assad, S. G. B. et al. (2017). Educação permanente em saúde e atividades de vacinação: revisão integrativa. Rev Enferm UFPE; 11(1):410-21.

Assad, S. G. B. et al. (2020). Permanent education and vaccination: minimizing missed opportunities. Research, Society and Development, [S. 1.], 9(11), p. e59391110198.

Bomfim, E. S. et al. (2017). Educação permanente no cotidiano das equipes de saúde da família: utopia, intenção ou realidade? Rev cuidado é fundamental Online (Rio de Janeiro). 9(2), 526-535, abr-jun.

Brasil. (2013). Ministério da Saúde (BR); Secretaria de Atenção à Saúde, Departamento de Ações Programáticas Estratégicas. Caderneta de Saúde da Criança: passaporte para a cidadania. Brasília: Ministério da Saúde.

Brasil. (2014). Ministério da Saúde (BR). Secretaria-Executiva. Subsecretaria de Assuntos Administrativos. Educação Permanente. p.120;

Brasil. (2017). Ministério da Saúde. Portaria n0 2436 de 21 de setembro de 2017. Brasília. Diário Oficial da República Federativa do Brasil.

Brasil. (2018). Ministério da Saúde. Secretaria de Gestão do Trabalho e da Educação na Saúde. Departamento de Gestão da Educação na Saúde. Política Nacional de Educação Permanente em Saúde: o que se tem produzido para o seu fortalecimento?

Bujes, M. K. (2012). Motivos do atraso vacinal em crianças - uma pesquisa bibliográfica. UFRGS.

Campos, K. F. C, Sena, R. R, \& Silva, K. L. (2017). Educação permanente nos serviços de saúde. Esc Anna Nery. 21(4):e20160317.

Cosme, F. S. M. N., \& Valente, G. S. C. (2020). Educação permanente na práxis de preceptoria em Atenção Básica de Saúde. Research, Society and Development, $9(8), 1-21$.

Cunha, A. Z. S. et al. (2014). Implicações da educação permanente no processo de trabalho em saúde. Rev Espaço Saúde.

Esperón, J.M.T. (2017) Pesquisa quantitativa na ciência da enfermagem. Esc. Anna Nery. 21(1): e20170027.

Fleury, M. T. L., \& Werlang, S. R.C. (2017). Pesquisa aplicada: conceitos e abordagens. Anuário de Pesquisa GV Pesquisa. 
Research, Society and Development, v. 10, n. 7, e30010716591, 2021

(CC BY 4.0) | ISSN 2525-3409 | DOI: http://dx.doi.org/10.33448/rsd-v10i7.16591

Godoi, B. B. et al. (2018). Capacitação de agentes comunitários de saúde no município de Diamantina/MG. Rev.Ciênc. Ext. 14(1), 54-69.

Guedes, M. B. O. G. et al. (2014). Capacitação dos agentes comunitários de saúde de uma unidade básica em Santa Cruz-RN: a extensão universitária em ação. Revista Extensão e Sociedade, 1(7), 1-13.

Lima, L. G. (2016). A Utilização da Caderneta de Saúde da Criança no acompanhamento Infantil. Rev Bras Cien Saúde. 20(2):167-74.

Maciazeki-Gomes, R. C. et al. (2016). O trabalho do agente comunitário de saúde na perspectiva da educação popular em saúde: possibilidades e desafios. Ciênc. saúde coletiva, 21(5), 1637-1646.

Maciel, F. B. M. et al. (2020). Agente comunitário de saúde: Reflexões sobre o processo de trabalho em saúde em tempos de pandemia de Covid-19. Cien Saude Coletiva. 25(Supl.2):4; 4185-4195.

Martins, J. R. T. et al. (2019). A vacinação no cotidiano: vivências indicam a Educação Permanente. Escola Anna Nery, 23(4), 1-8.

Merhy, E. E. (2015). Educação permanente em movimento: uma política de reconhecimento e cooperação, ativando os encontros do cotidiano no mundo do trabalho em saúde, questões para os gestores, trabalhadores e quem mais quiser se ver nisso. Saúde em Redes. 1(1):7-14.

Musse, J. O. et al. (2015). Avaliação de competências de agentes comunitários de saúde para coleta de dados epidemiológicos. Ciência e Saúde Coletiva, 20(2), 525-536.

Santos, K. T. et al. (2011). Agente comunitário de saúde: perfil adequado a realidade do Programa Saúde da Família? Ciênc. saúde coletiva, 16(supl 1), 10231028.

Santos, W. J. et al. (2020). Avaliação do conhecimento de Agentes Comunitários de Saúde sobre o conteúdo da Caderneta da Saúde da Criança. Journal of Health \& Biological Sciences, 8(1), 1-5.

Secco, A. C. et al. (2020). Educação Permanente em Saúde para Agentes Comunitários: um Projeto de Promoção de Saúde. Gerais, Rev. Interinst. Psicol., Belo Horizonte, 13(1), 1-17, jan.

Silva, F. B., \& Gaíva, M. A. M. (2016). Dificuldades enfrentadas pelos profissionais na utilização da caderneta de saúde da criança. Rev Bras Pesq Saúde. 18(2): 96-103.

Sociedade Brasileira De Pediatria. (2017). Departamento Científico de Pediatria do Desenvolvimento e Comportamento. Guia Prático de Atualização. Caderneta de Saúde da Criança e do Adolescente: instrumentos de vigilância e promoção do Desenvolvimento. 4:1-5.

Tomaz, J. B. C. (2002). O agente comunitário não deve ser um “super-herói”. Interface. Comunicação, Saúde e Educação. 6(10):84-87. 